\title{
Calreticulin from suboolemmal vesicles affects membrane regulation of polyspermy
}

\author{
María Dolores Saavedra, Irene Mondéjar, Pilar Coy , Miguel Betancourt, \\ Humberto González-Márquez, María Jiménez-Movilla ${ }^{2}$, Manuel Avilés ${ }^{2}$ and Raquel Romar ${ }^{1}$ \\ Department of Health Science, Metropolitan Autonomous University, Campus Iztapalapa, 09340 Mexico DF, Mexico, \\ ${ }^{1}$ Department of Physiology, Campus of International Excellence 'Campus Mare Nostrum', Instituto Murciano de \\ Investigación Biosanitaria, University of Murcia, Murcia, Spain and ${ }^{2}$ Department of Cell Biology and Histology, \\ Campus of International Excellence 'Campus Mare Nostrum', Instituto Murciano de Investigación Biosanitaria, \\ University of Murcia, Murcia, Spain \\ Correspondence should be addressed to R Romar; Email: rromar@um.es
}

\begin{abstract}
This study was designed to determine whether calreticulin (CRT), a chaperone protein, is present in in vitro-matured (IVM) pig oocytes and to study its potential role in the block to polyspermy. Western blot analysis, using an anti-CRT antibody, of oocyte lysate showed an immunoreactive band of $\sim 60 \mathrm{kDa}$. Simultaneous labeling of IVM oocytes with anti-CRT antibody and peanut agglutinin lectin (PNA lectin, a porcine cortical granules (CG)-specific binding lectin) revealed localization of CRT in the subplasmalemmal region with a $27.7 \%$ colocalization with PNA staining. After IVF, PNA labeling was not observed and anti-CRT labeling decreased significantly in zygotes and disappeared in two-cell embryos. Western blot analysis of oocyte exudate obtained from zona pellucida (ZP)-free oocytes activated with calcium ionophore confirmed the presence of a band that reacted with an anti-CRT antibody. Anti-CRT antibody and PNA labeling were not observed in activated oocytes despite being detectable in non-activated oocytes. The presence of CRT in vesicles located under the oolemma was demonstrated using immunogold cytochemistry at the ultrastructural level. To study the role of CRT in fertilization, ZP-enclosed and ZP-free oocytes were incubated with exogenous CRT and then inseminated. Whereas ZP-free oocytes showed fewer penetrating sperm and lower polyspermy rates than untreated oocytes, the opposite effect was observed in ZP-enclosed oocytes. In conclusion, CRT is confined to subplasmalemmal vesicles partially overlapping with CG contents. Its exocytosis after the oocyte activation seems to participate in the membrane block to polyspermy in pigs but is not involved in the ZP block.

Reproduction (2014) 147 369-378
\end{abstract}

\section{Introduction}

Following gamete membrane fusion at fertilization, the cortical granules (CG) undergo exocytosis releasing their contents into the perivitelline space, modifying the egg's zona pellucida (ZP), and preventing penetration by additional spermatozoa (reviewed by Wessel et al. (2001)). Although the specific CG components responsible for the zona block have not yet been identified, several proteins have been partially characterized and reported to be released from CG or present in the medium surrounding activated eggs (exudate). Mouse CG contain ovoperoxidase (Gulyas \& Schmell 1980), $\mathrm{N}$-acetylglucosaminidase (Miller et al. 1993), peptidylarginine deiminase (p75) (Pierce et al. 1992, Liu et al. 2005), a 32-kDa protein named P32 (Gross et al. 2000), several proteases (Moller \& Wassarman 1989, Tawia \& Lopata 1992, Burkart et al. 2012), and glycoconjugates (Lee et al. 1988). Carbohydrates are present in human CG
(Jiménez-Movilla et al. 2004); hamster CG contain two proteins (P56 and P62) immunologically related to sea urchin hyaline (Hoodbhoy et al. 2000); it has been suggested that rat CG contain tissue plasminogen activator (Zhang et al. 1992) and that this protein is also present in bovine CG (Rekkas et al. 2002), but ultrastructural studies have demonstrated its presence in the oolemma and not in the CG (Mondéjar et al. 2012). Functions of CG-derived proteins have been related to ZP modifications and further block to polyspermy (Moller \& Wassarman 1989, Tawia \& Lopata 1992, Zhang et al. 1992, Miller et al. 1993, Wessel et al. 2001), and regulation of preimplantation cleavage and early embryonic development (Hoodbhoy \& Talbot 2001, Hoodbhoy et al. 2001). Studies concerning CG contents in pig oocytes are scarce and no specific granule protein has been described. Polyspermy remains an unsolved problem in the current IVF systems (Coy \& Avilés 2010). Porcine CG contain glycoconjugates with terminal 
$\beta$-D-galactosyl (1,3)-D- $N$-acetylgalactosamine (Gal $\beta 1-$ 3GalNAc) residues, and peanut agglutinin lectin (PNA lectin) is the only specific available tool for labeling pig CG contents (Yoshida et al. 1993).

More recently, attention has been drawn to calreticulin (CRT), an ancient highly conserved ubiquitous protein originally identified as an endoplasmic reticulum chaperone (Ostwald \& MacLennan 1974). This protein contains an $\mathrm{N}$-terminal cleavable signal sequence that directs it to the endoplasmic reticulum (ER), and an ER KDEL (Lys-Asp-Glu-Leu) retention/retrieval signal. When residing in the ER, CRT has important functions in directing proper folding of proteins and glycoproteins, as well as in homeostatic control of cytosolic and endoplasmic reticulum $\mathrm{Ca}^{2+}$ levels. There is also steadily accumulating evidence of diverse roles for CRT localized outside the ER, including data suggesting important roles for CRT localized on the outer cell surface of a variety of cell types, in the cytosol, and in the extracellular matrix (reviewed by Gold et al. (2010)).

CRT is the major $\mathrm{Ca}^{2+}$-binding chaperone and it has been linked to CG being localized in the cortical area of human eggs (Balakier et al. 2002), hamster CG (Muñoz-Gotera et al. 2001), and on the extracellular surface of the mouse egg oolemma remaining in the perivitelline space after egg activation (Tutuncu et al. 2004). Confocal studies in porcine oocytes by Zhang et al. (2010) identified CRT at a subcellular level accumulated primarily in the cortical/plasma membrane region of matured oocytes. They associated CRT with the regulation of oocyte meiotic maturation and maternal gene expression but did not compare CRT localization with that of CG or other subplasmalemmal vesicles. A specific function of CRT on fertilization or postfertilization events in mammals has not been fully described, although it has been associated with the block to polyspermy by remaining in the perivitelline space after being exocytosed (Muñoz-Gotera et al. 2001). Recent studies show that topically applied CRT has diverse and profound biological effects that enhance cutaneous wound healing in animal models (Gold et al. 2010). This evidence for extracellular bioactivities of CRT has provided new insight into this classically ER-resident protein, despite a lack of knowledge on how CRT progresses from the ER to the cell surface or how it is released into the extracellular milieu. Nonetheless, it has become clear that CRT is a multicompartmental protein that regulates a wide array of cellular responses (Gold et al. 2010).

Is CRT located in the pig CG and, if so, does it have a role in the block to polyspermy? Our first hypothesis is that CRT is contained in CG. To confirm this, oocyte lysate and exudate from in vitro-matured (IVM) oocytes was analyzed by western blot with an anti-CRT antibody. CRT protein was immunolocalized in IVM oocytes, zygotes, and two-cell embryos, and transmission electron microscopy (TEM)-immunogold showed the detailed location of the protein. Our second hypothesis is that CRT participates somehow in the block to polyspermy; this was tested by inseminating ZP-free and ZP-enclosed oocytes previously treated with exogenous CRT.

\section{Materials and methods}

This study was developed following institutional approval from the Bioethical Committee in the University of Murcia and was performed in accordance with the Animal Welfare regulations of that institution (Permit for study number: AGL2009-12512C02-01). All the chemicals and reagents were purchased from Sigma-Aldrich unless otherwise stated.

\section{Oocyte collection and IVM}

Cumulus-oocyte complexes (COCs) were collected by aspiration of antral follicles (3-6 mm diameter) of ovaries from slaughtered 6- to 7-month-old animals (Landrace X Large White), weighing $80-100 \mathrm{~kg}$. Only COCs with a dark cytoplasm and a complete and dense cumulus oophorus were used for the experiments. Groups of 50-55 COCs were IVM for $44 \mathrm{~h}$ in NCSU-37 medium supplemented with $0.57 \mathrm{mM}$ cysteine, $1 \mathrm{mM}$ dibutyryl cAMP, $5 \mu \mathrm{g} / \mathrm{ml}$ insulin, $50 \mu \mathrm{M} \beta$-mercaptoethanol, $1 \mathrm{mM}$ glutamine, $10 \mathrm{lU} / \mathrm{ml}$ eCG (Folligon, Intervet International B.V., Boxmeer, Holland), $10 \mathrm{IU} / \mathrm{ml}$ hCG (Veterin corion, Divasa Farmavic, Barcelona, Spain), and $10 \%(\mathrm{v} / \mathrm{v})$ porcine follicular fluid (Funahashi et al. 1997, Romar et al. 2005).

\section{Collection of oocyte lysate and exudate}

Oocyte lysate was obtained from matured COCs as follows. After IVM, COCs were stripped of cumulus cells by pipetting in PBS and those showing extrusion of the first polar body were quickly washed three times in ultrapure water (MilliQ, Millipore, Billerica, MA, USA), transferred to a water droplet, at a ratio of 200 oocytes in $50 \mu \mathrm{l}$, and allowed to lyse until the full release of cytoplasmic content (Supplementary Figure 1, see section on supplementary data given at the end of this article). All processes were carried out at $4{ }^{\circ} \mathrm{C}$ and, when necessary, oocytes were passed through a narrow glass pipette of $\sim 80 \mu \mathrm{m}$ diameter to accelerate the release of cytoplasmic content. Once lysed, the ZPs were removed from the water droplet and washed three times in fresh water droplets. All droplets containing the cytoplasmic content (lysate) and ZPs were quickly aspirated separately and stored at $-80^{\circ} \mathrm{C}$ until use.

Oocyte exudate was obtained from ZP-free activated oocytes. IVM oocytes were mechanically denuded, ZP was digested with acidic Tyrode's solution (Sigma T1788), and ZP-free oocytes were washed three times in PBS without $\mathrm{Ca}^{2+}-\mathrm{Mg}^{2+}$. Then, ZP-free oocytes were incubated with calcium ionophore A23187 $(6.5 \mu \mathrm{M}, 2 \mathrm{~min})$, washed three times in a medium consisting of $114.06 \mathrm{mM} \mathrm{NaCl}, 3.2 \mathrm{mM}$ $\mathrm{KCl}, 0.5 \mathrm{mM} \mathrm{MgCl} 2 \cdot 6 \mathrm{H}_{2} \mathrm{O}, 10 \mathrm{mM}$ sodium lactate, $0.35 \mathrm{mM}$ $\mathrm{NaH}_{2} \mathrm{PO}_{4}, 5 \mathrm{mM}$ glucose, $25.07 \mathrm{mM} \mathrm{NaHCO}$, $8 \mathrm{mM}$ calcium 
lactate $5 \mathrm{H}_{2} \mathrm{O}(\mathrm{pH} 7.2,320 \mathrm{mOsm})$, and transferred to a $50 \mu \mathrm{l}$ droplet of fresh medium. Activated ZP-free oocytes were incubated at $38.5{ }^{\circ} \mathrm{C}$ and $5 \% \mathrm{CO}_{2}$ for $30 \mathrm{~min}$ to allow CG content release. After this time, ZP-free oocytes were carefully aspirated, a portion lysed as described previously, and the other portion processed to assess cortical reaction under confocal and TEMs. The medium containing the oocyte's exudate was collected, stored at $-80{ }^{\circ} \mathrm{C}$, and further used for electrophoresis and western blot.

\section{Western blot analysis}

CRT rabbit polyclonal antibody (synthetic peptide QAKDEL corresponding to $\mathrm{C}$ terminal amino acids of human CRT) was purchased from Abcam, Cardiff, UK (ab15607). The proteins in the oocyte lysate (both from IVM oocytes and from ZP-free activated oocytes) and exudate were dissolved in reducing SDS-PAGE loading buffer, separated by SDS/PAGE electrophoresis (RT, $1 \mathrm{~h}$ at $150 \mathrm{~V}$ ) and electrotransferred to polyvinylidene fluoride membrane $\left(4^{\circ} \mathrm{C}, 1 \mathrm{~h}\right.$ at $\left.100 \mathrm{~V}\right)$. The membranes were washed and blocked in Tris-buffered saline (TBS) with $0.1 \%$ Tween 20 and $2 \%$ BSA at $37{ }^{\circ} \mathrm{C}$ for $1 \mathrm{~h}$ and incubated with anti-CRT antibody $(1: 1000)$ at $37^{\circ} \mathrm{C}$ for $1 \mathrm{~h}$. Then the membrane was washed three times with TBS with $0.1 \%$ Triton X-100 (TBST) and incubated with HRP-conjugated secondary antibody (Santa Cruz Biotechnology, sc2004; $1: 20000)$ at $37^{\circ} \mathrm{C}$ for $1 \mathrm{~h}$. After washing in TBST, the protein binding was detected using the $\mathrm{ECL}$ plus method and Typhoon 9410 (Amersham Biosciences). Briefly, the membrane was incubated for $5 \mathrm{~min}$ with the chemifluorescence $\mathrm{ECL}$ Plus substrate (40:1 mixture of component $A$ and $B$ ) as instructed by the product manual. Then the membrane was drained, scanned with the appropriate filters for HRP (excitation 430 and $503 \mathrm{~nm}$ emission), and the obtained images saved as computer files.

To discard that CRT or cytoplasmic content had been aspirated with the ZPs, the samples $(n=200)$ were solubilized $\left(70{ }^{\circ} \mathrm{C}, 30 \mathrm{~min}\right)$, dissolved in SDS-PAGE loading buffer, and processed as described above. Human CRT full-length protein (ab91577) was used as control $(0.1 \mu \mathrm{g})$. Anti- $\beta$-actin antibody (Abcam ab8226) was used as marker of cytoplasmic content using boar spermatozoa lysate $(20 \mu \mathrm{g})$ as control.

\section{IVF and embryo culture}

The fertilization medium was modified TALP supplemented with $3 \mathrm{mg} / \mathrm{ml}$ fatty acid-free BSA and $1.10 \mathrm{mM}$ sodium pyruvate (Rath \& Niemann 1997). After IVM, COCs were denuded and transferred to a four-well multidish containing $250 \mu \mathrm{I}$ IVF medium previously equilibrated at $38.5^{\circ} \mathrm{C}$ under $5 \% \mathrm{CO}_{2}$. A sperm-rich fraction of semen from a mature, fertility-tested boar was collected and semen samples $(0.5 \mathrm{ml})$ were centrifuged (700 $\mathbf{g}, 30 \mathrm{~min}$ ) through a discontinuous Percoll (Pharmacia) gradient (45 and 90\% v/v). Resultant sperm pellets were diluted in fertilization medium and centrifuged again (10 min, $100 \mathrm{~g}$ ). Finally, the pellet was diluted in fertilization medium and $250 \mu$ of this suspension were added to the wells containing the oocytes, giving a final concentration of $10^{5}$ cells $/ \mathrm{ml}$. An aliquot of zygotes was processed for immunocytochemistry at $18 \mathrm{~h}$ post-insemination (hpi) and those remaining were cultured for an additional $24 \mathrm{~h}$ in NCSU-23 medium up to the two-cell embryo stage.

\section{Immunofluorescence labeling of oocytes, zygotes, and embryo for CRT and protein disulphide isomerase localization}

CRT antibody (Abcam ab15607) was used in combination with a secondary antibody conjugated with rhodamine isothiocyanate (anti-rabbit IgG-TRITC; 1:400) purchased from Sigma (T6778). The protein disulphide isomerase (PDI) mouse antibody (clone 1D3 corresponding to the sequence near the C-terminus of rat PDI) was purchased from Enzo Life Sciences, Lausanne, Switzerland (ADI-SPA-891) and was used in combination with a secondary antibody conjugated with FITC (anti-mouse IgG-FITC; 1:400) purchased from Millipore (12-506). Immunofluorescence staining of proteins was done as described previously (Mondéjar et al. 2012). Briefly, samples were first fixed in $3.7 \%$ freshly prepared paraformaldehyde in PBS $(30 \mathrm{~min}$ ) followed by permeabilization in $0.1 \%$ Triton X-100 (10 min) and blocking with $1 \%$ BSA and $100 \mathrm{mM}$ glycine $(30 \mathrm{~min})$. Then, samples were incubated for $1 \mathrm{~h}$ in the dark at room temperature with CRT $(1: 10)$ or PDI $(1: 1000)$ primary antibodies followed by incubation with their corresponding secondary antibodies for $1 \mathrm{~h}$. Finally, samples were labeled with PNA lectin conjugated to FITC $(10 \mu \mathrm{g} / \mathrm{ml}, 30 \mathrm{~min})$ to specifically label CG (Yoshida et al. 1993). After antibody and lectin incubations, samples were washed three times in equilibration buffer medium (Slow fade, Antifade kit, Molecular Probes, Eugene, OR, USA), mounted and kept in the dark at $4{ }^{\circ} \mathrm{C}$ until assessment within $24 \mathrm{~h}$ using confocal laser scanning microscopy (Nikon Eclipse). As negative controls, samples were incubated only with the secondary antibody. CRT protein was detected in oocytes $(n=20)$, zygotes $(n=15)$, and two-cell embryo $(n=10)$ and PDI protein was detected in oocytes $(n=15)$ and zygotes $(n=15)$ from three independent experiments. Images were sequentially acquired in order to study the degree of overlapping between FITC (CG labeling) and TRITC (CRT labeling). Both equatorial and cortex images from each oocyte were used to calculate Pearson's correlation and Manders' colocalization coefficients using a colocalization software (NIS Elements Microscope Imaging Software, Tokyo, Japan). The degree of overlapping between two different fluorescent labels, each having a separate emission wavelength, is used to discriminate whether two different cellular targets are located in the same area or very near to each other (Zinchuk \& Zinchuk 2008).

Images were also analyzed to quantify the intensity of antiCRT labeling by means of the free software ImageJ (http:// rsbweb.nih.gov/ij/). Briefly, images captured under RGB format were transformed into an eight-bit gray image and segmented to delimit the labeled area under a fixed threshold for gray color (values from 1 to 255). The generated binary mask of each image was used to measure the area (square micrometers) and the labeling intensity (mean gray color of the pixels in the area of interest) in the oocyte's cortical area and ZP. An example of the image analysis is presented in 
Supplementary Figure 2, see section on supplementary data given at the end of this article.

\section{CG quantification by TEM}

IVM oocytes $(n=18)$ and ZP-free activated oocytes $(n=12)$ from three independent experiments were fixed in $2 \%$ glutaraldehyde in 0.1 M PIPES buffer $\left(\mathrm{pH} \mathrm{7.2)}\right.$ for $1 \mathrm{~h}$ at $4{ }^{\circ} \mathrm{C}$ and post-fixed in $2 \%$ osmium tetroxide at room temperature. For preparation of ultrathin sections (50 nm thickness), oocytes were processed for embedding in Embed 812 (EMC, Washington, Pennsylvania 19034, USA), sections obtained with an ultramicrotome (Microm International $\mathrm{GmbH}$, Walldorf, Germany), and mounted on formvar-coated nickel grids. After washing twice in distilled water, the grids were counterstained with uranyl acetate and lead citrate. The thin sections were observed under a Phillips Tecnai 12 electron microscope (Eindhoven, The Netherlands). For quantitative analysis of CG, four images at $2850 \times$ were taken randomly in each oocyte and number of CG further counted.

\section{TEM immunogold of oocytes and zygotes for CRT and PDI localization}

For TEM, a rabbit polyclonal anti-CRT antibody tested for electron microscopy was purchased from Abcam (ab4) and used at 1:10 dilution. Anti-PDI antibody from Enzo Life Sciences (ADI-SPA-891) was used at $2 \mu \mathrm{g} / \mathrm{ml}$. Samples were fixed with $1 \%$ glutaraldehyde in cacodylate buffer $(\mathrm{pH} 7.4)$ for $2 \mathrm{~h}$ at $4{ }^{\circ} \mathrm{C}$. After extensive washing in cacodylate buffer, samples were dehydrated through a graded series of ethanol and processed for embedding in LR-White resin. For immunocytochemistry, a two-step method was used. Ultrathin sections of oocytes and zygotes were incubated with anti-CRT and oocytes also with anti-PDI antibody. Grids were then washed and floated on a drop of Protein A gold (15 nm) conjugated antibody (Utrecht University; 1:60). After washing twice in distilled water, the grids were counterstained and observed as mentioned in the above section.

\section{IVF of ZP-free and ZP-enclosed mature oocytes incubated with exogenous $C R T$}

A recombinant full-length human CRT was purchased from Abcam (ab91577). Matured COCs were denuded by pipetting and half of the oocytes were subjected to ZP digestion in acidic Tyrode's solution. ZP-free and ZP-enclosed oocytes were incubated for $30 \mathrm{~min}$ in fertilization medium supplemented with $0,100,1000$, and 5000 pg of CRT per oocyte. Protein incubation time was chosen based on preliminary trials from our laboratory and the literature (Tutuncu et al. 2004). After CRT treatment, oocytes were gently washed in fertilization medium and inseminated as described in the above section with a final sperm concentration of $10^{5}$ cells/ml (ZP-enclosed oocytes) or 2.500 cells/ml (ZP-free oocytes). At $18 \mathrm{hpi}$, putative zygotes were fixed, stained (Hoechst 33342), and sperm penetration, number of spermatozoa per penetrated oocyte, male pronucleus formation, and number of sperm bound to the ZP or oolemma were assessed.

\section{Statistical analysis}

Data are presented as the mean \pm S.E.M. and all percentages were modeled according to the binomial model of variables. The IVF variables were analyzed by one-way ANOVA (percentage of oocyte penetration, mean number of sperm cells per penetrated oocyte, percentage of male pronucleus formation, percentage of monospermy, and number of sperm bound to the ZP or oolemma). When ANOVA revealed a significant effect, values were compared by Tukey's test. A $P$ value $<0.05$ was considered to denote statistical significance.

\section{Results}

\section{CRT is present in vesicles in the cortex of matured pig oocytes partially colocalizing with CG contents}

Western blot of the lysate obtained from IVM oocytes showed an immunoreactive band of $\sim 60 \mathrm{kDa}$, consistent with the expected size of CRT (Fig. 1A). In the samples from activated oocytes, no immunoreactive band was observed in the lysate but was present in the exudate, confirming that the band corresponds to the soluble isoform of the protein. The western blot of the ZPs showed that no cytoplasmic content was aspirated with them. Neither calretinin nor actin is detected in the ZPs aspirated from the lysis droplets (Fig. 1B).

Indirect immunofluorescence demonstrated that CRT protein is located within the cortical region of metaphase II oocytes (Fig. 2A) with a mean intensity labeling of $30.3 \pm 0.2$ units/area. The negative controls incubated with only the secondary antibody did not show any signal (image not shown). A defined CG band was present in IVM oocytes after labeling with PNA, which specifically binds to these organelles (Fig. 2B). Dualchannel images obtained by sequential scanning showed overlapping between FITC and TRITC fluorochromes, meaning that PNA and CRT colocalize to some extent in the same oocyte (Fig. 2C). Results showed a Mander's coefficient of 0.277 and a Pearson's correlation coefficient of 0.61. Mander's index indicates that $27.7 \%$ of the pixels in the image had overlapping of FITC and TRITC fluorophores whereas Pearson's coefficient ranges from -1.0 to 1.0 and values over 0.5 indicate that the colocalization is significant (Zinchuk \& Zinchuk 2008).

At the light resolution limits of confocal microscopy, the immunofluorescent signals at the cortex can originate from either membrane proteins that reside at the oolemma or at the secretory vesicles that are close to the oolemma. To confirm that anti-CRT labeling did not originate from the oolemma itself, non-permeabilized oocytes were incubated with antiCRT antibody. Results showed a very weak anti-CRT staining and not a full cortical labeling pattern (Supplementary Figure 3, see section on supplementary data given at the end of this article). 


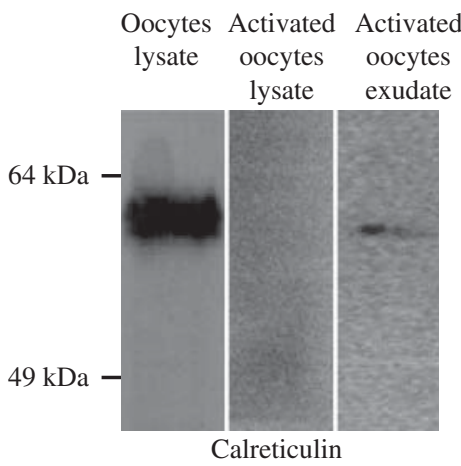

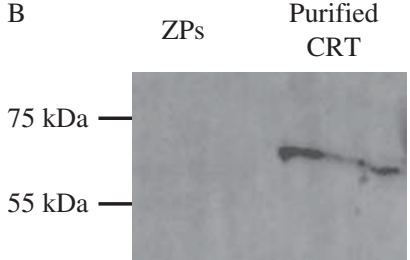

Calreticulin

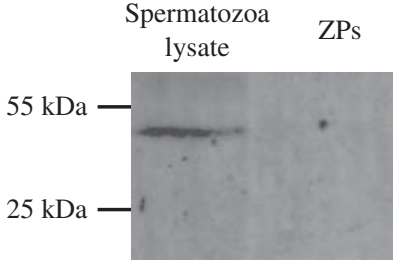

Actin
Figure 1 (A) Calreticulin (CRT) is present in the lysate and exudate collected from mature pig oocytes. Lysate is the whole cytoplasmic content of mature oocytes obtained after being lysed in water. Exudate corresponds to the content exocytosed after activation of mature oocytes. Western blots of lysate obtained from in vitro-matured oocytes $(n=150)$, lysate from ZP-free oocytes activated with calcium ionophore $(n=250)$, and the collected exudate. Experiment was replicated three times. (B) Cytoplasmic content is not detected in the ZPs. Neither calretinin nor actin is detected in the ZPs $(n=200)$ aspirated from lysis droplets. Controls used for the ZP western blots are human CRT full-length protein $(0.1 \mu \mathrm{g})$ and boar spermatozoa lysate $(20 \mu \mathrm{g})$.

The specific localization of CRT in vesicles located immediately adjacent to the oolemma was demonstrated using immunogold cytochemistry at the ultrastructural level (Fig. 3). CRT localization was partially associated with CG and other cortical vesicles. The images obtained by TEM showed that CRT and CG are located close to each other in the egg cortex. After fertilization, CG and CRT molecules were exocytosed from the egg cortex and some CRT molecules were observed at the oolemma and ZP. These observations open the possibility that $\mathrm{CRT}$, a classically ER-resident protein, was contained in secretory vesicles distinct from CG, which would be exocytosed after oocyte activation. To confirm this hypothesis, oocytes and zygotes were immunolabeled with a specific anti-PDI antibody, a classic ER-marker. Samples were observed under confocal microscopy and TEM to study the localization and likely exocytosis of ER-derived vesicles. Results showed that after IVM, PDI protein was present in vesicles located in the cortex of matured oocytes in a similar distribution pattern than CG (Supplementary Figure 4, see section on supplementary data given at the end of this article). After IVF, the anti-PDI labeling disappeared and was no longer observed. These observations indicate a release into the extracellular milieu and might suggest extracellular bioactivities of CRT and PDI.

\section{CRT is exocytosed after the cortical reaction}

Confocal visualization of zygotes showed that CG had been fully exocytosed as demonstrated by the disappearance of the CG band from the cortical region (Fig. 4). Anti-CRT staining of zygotes at the cortical level almost fully disappeared, reaching an intensity of $1.8 \pm 0.2$ units/area $(n=10)$, a $94 \%$ reduction compared with unfertilized oocytes. At the resolution of confocal imaging, no anti-CRT antibody labeling was observed
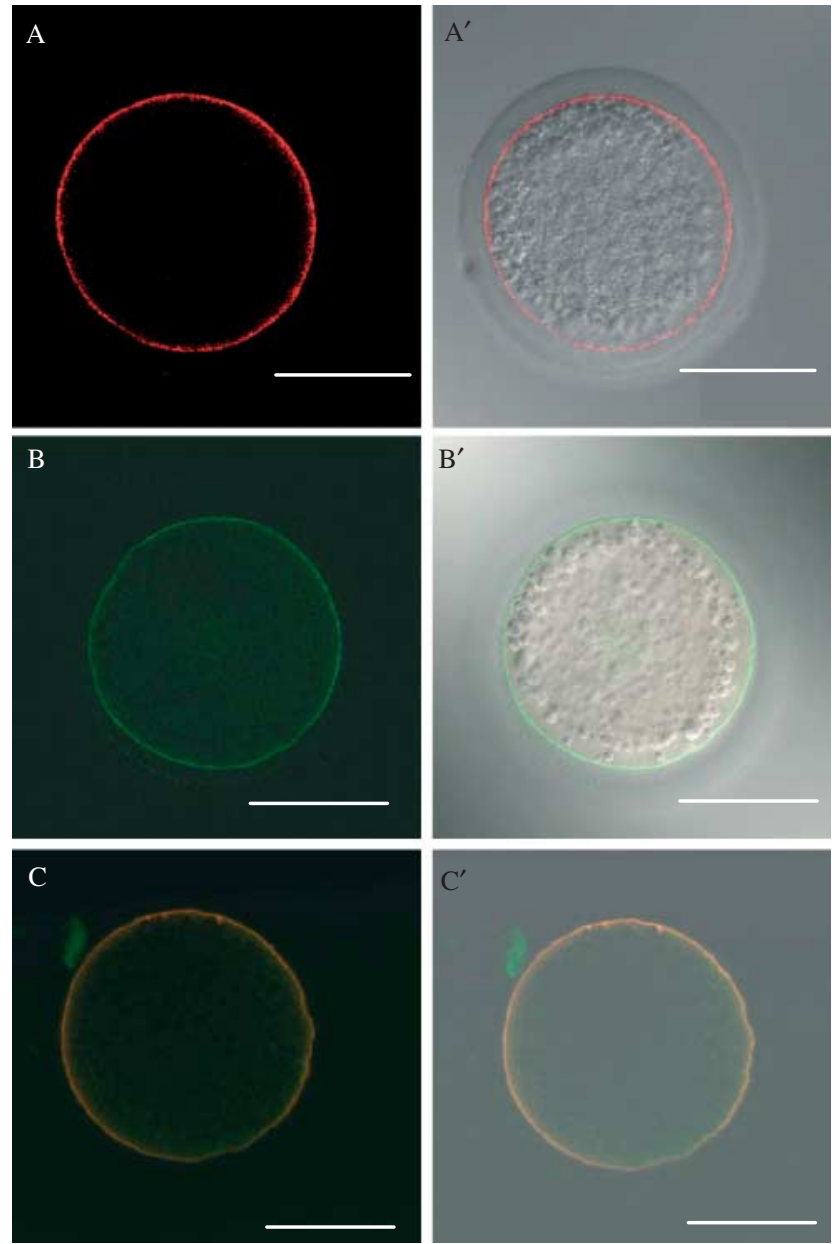

Figure 2 Calreticulin (CRT) is located in the cortex region of ZP-enclosed mature pig oocytes. Confocal visualization of CRT and cortical granules (CG) in in vitro-matured pig oocytes. (A and $\mathrm{A}^{\prime}$ ) Immunofluorescent labeling of CRT protein (red) showing intense fluorescence in the cortical region. (B and $\left.\mathrm{B}^{\prime}\right)$ Staining with peanut agglutinin lectin conjugated to fluorescein (PNA-FITC) (green) indicates a defined cortex arrangement of CG adjacent to oolemma. (C and $\mathrm{C}^{\prime}$ ) Colocalization (overlapping) between PNA-FITC and anti-CRT antibody with a secondary antibody conjugated to rhodamine (CRT-TRITC) in the entire cortical area of the oocytes. Bar represents $50 \mu \mathrm{m}$. 


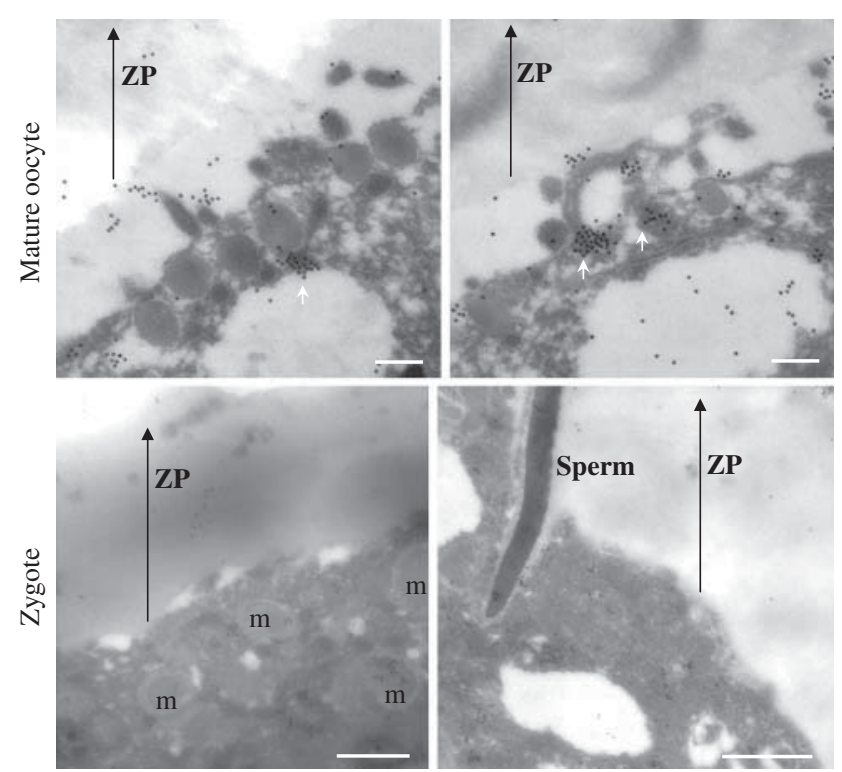

Figure 3 Calreticulin (CRT) is present in IVM oocytes and exocytosed in zygotes. (Mature oocytes) Transmission electron microscopyimmunogold demonstration of CRT (black dots) in in vitro-matured pig oocytes. Arrows indicate CRT molecules close to or associated with cortical granules (CG) and other subplasmalemmal vesicles in the egg cortex. CRT is absent from the zona pellucida (ZP). Bar: $0.2 \mu \mathrm{m}$ (Zygotes) CG and CRT molecules are exocytosed from egg cortex. $A$ residual CRT is observed on the oolemma and ZP. $m$, mitochondrion. Bar represents $1 \mu \mathrm{m}$.

in the perivitelline space of zygotes and labeling at ZP level was minimal with $1.5 \pm 0.2$ units/area (a $95 \%$ reduction compared with unfertilized oocytes) coinciding with previous observations by electron microscopy (Fig. 3). No anti-CRT antibody labeling was observed in the blastomeres of two-cell embryos whereas the ZP showed a residual intensity of $2.1 \pm 0.1$ units/area (Fig. 4). A positive PNA staining was observed on the outer ZP of zygotes and two-cell embryos due to the presence of acrosomal shrouds (Romar et al. 2012).

Cortical reaction induced in ZP-free IVM oocytes by calcium ionophore ( $6.5 \mu \mathrm{M}$ for $2 \mathrm{~min}$ and further $30 \mathrm{~min}$ incubation) was confirmed by confocal microscopy and TEM since no literature was found about the CG exocytosis degree after chemical activation of porcine ZP-free oocytes. Results showed that ZP removal did not affect the integrity of the oolemma, and the CG remained tightly located near the plasma membrane (Fig. 5) with a mean number of $5.9 \pm 1.8 \mathrm{CG}$ per $5 \mu \mathrm{m}$ of oolemma. This number significantly decreased to $0.7 \pm 0.2$ CG per $5 \mu \mathrm{m}$ of oolemma in activated oocytes. Thus our activation protocol with a 30-min post-activation period was efficient enough to allow CG exocytosis and to collect CG-derived proteins in the exudate.

Cytochemical localization showed that PNA (CG) and anti-CRT antibody labeling were present in the cortical region just after IVM and ZP removal (Fig. 6); however, this staining disappeared after oocyte activation. These observations are consistent with the appearance of an immunoreactive band for CRT in the immunoblot of exudate from activated oocytes as was shown in Fig. 1.

Taken together, these results strongly suggest that CRT is contained in secretory vesicles located within the oocyte's cortical area and is exocytosed during oocyte activation. The release into the extracellular milieu suggests an extracellular function for this protein, which was confirmed in the next experiment.

\section{CRT blocks polyspermy at oolemma level}

When ZP-enclosed oocytes were incubated with exogenous CRT before insemination, penetrability increased and monospermy was reduced (Table 1). Sperm penetration increased along with the number of sperm bound to the ZP compared with untreated ZP-enclosed oocytes. However, ZP-free oocytes incubated with CRT showed higher monospermy rates than untreated ZP-free oocytes. The increase in monospermy was not associated with a reduced penetration rate nor with a decrease in the number of sperm attached to the oolemma. Male pronucleus formation was not affected by CRT incubation and was over 95\% in all groups of oocytes.

\section{Discussion}

CRT has been associated with human eggs (Balakier et al. 2002) and porcine oocytes (Ellederova et al. 2004), hamster CG (Muñoz-Gotera et al. 2001) and oolemma,
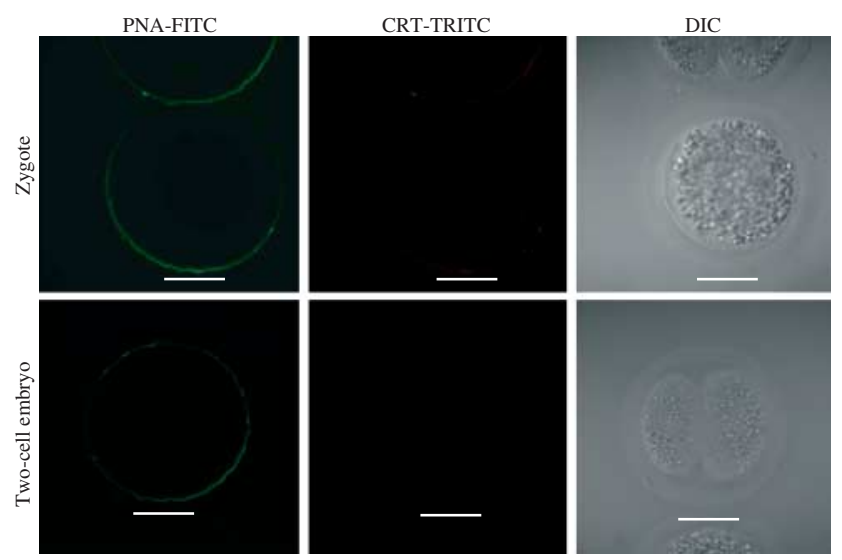

Figure 4 Calreticulin (CRT) and cortical granules labeling disappear in pig zygotes and embryos. Simultaneous labeling with anti-CRT antibody with a secondary antibody conjugated to rhodamine (CRT-TRITC) and peanut agglutinin lectin conjugated to fluorescein (PNA-FITC). Confocal visualization of zygotes obtained after IVF and assessed at $18 \mathrm{~h}$ post-insemination showed how PNA labeling disappeared from oolemma and anti-CRT labeling decreased significantly compared with oocytes. In the two-cell embryos assessed at $48 \mathrm{~h}$ post-insemination, both PNA and anti-CRT have completely disappeared. DIC, differential interference contrast. Bar represents $50 \mu \mathrm{m}$. 


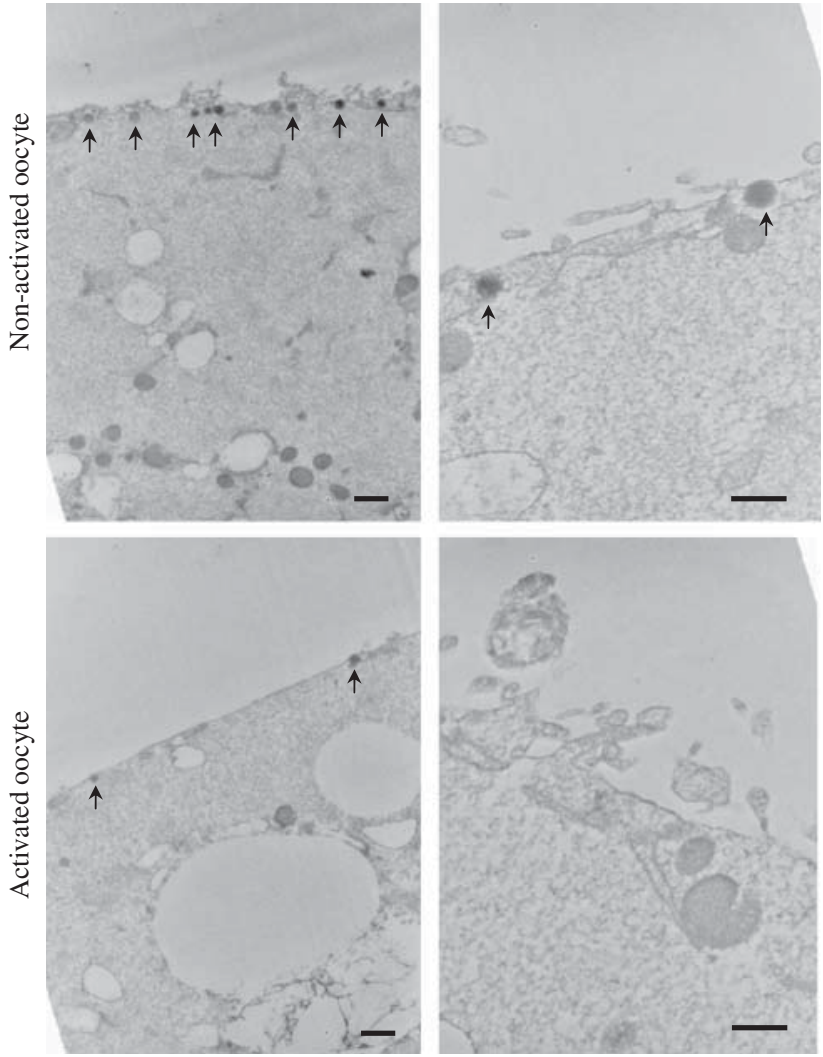

Figure 5 Cortical granules (CG) released after activation of ZP-free mature pig oocytes. Transmission electron microscopy images of in vitro-matured pig oocytes after ZP digestion with acidic Tyrode's solution showing CG (arrows) and oolemma integrity. After chemical activation with $6.5 \mu \mathrm{M}$ calcium ionophore for 2 min followed by 30 min incubation, the number of CG significantly decreased. Bar represents $1 \mu \mathrm{m}$.

and the perivitelline space of mouse eggs (Tutuncu et al. 2004) demonstrating several extracellular bioactivities (Gold et al. 2010) including the block to polyspermy in hamsters (Muñoz-Gotera et al. 2001). In this study, an anti-CRT antibody specifically recognized a single band of $60 \mathrm{kDa}$ in the lysate from IVM porcine oocytes. Conversely, CRT was not found in the lysate of ZP-free activated oocytes, but it appeared in the exudate collected from these oocytes, indicating that CRT had been exocytosed after oocyte activation. Protein bands observed in the immunoblots were consistent with the expected size of this protein coinciding with the molecular weight described in studies with mouse oocytes (60 kDa) (Tutuncu et al. 2004), protein extracts from hamster oocytes $(60 \mathrm{kDa})$ (Muñoz-Gotera et al. 2001), Xenopus oocytes (61 kDa) (Parys et al. 1994), and human oocytes (63 kDa) (Balakier et al. 2002).

The results from this study show that CRT is located in the cortical region of IVM porcine oocytes, associated with secretory vesicles and partially colocalizing with CG contents. CRT is released after induction of cortical reaction both by spermatozoa (physiological induction) and calcium ionophore (artificial induction). CRT signal is then weakly observed on the oolemma of zygotes and is no longer observed in two-cell embryos. These results are consistent with a recent study in pigs reporting the accumulation of CRT in cortical/plasma membrane region of metaphase II oocytes with a weak fluorescence signal in the cytoplasm of pronuclear-stage zygotes and early cleaved embryos (Zhang et al. 2010). The CRT intracellular localization pattern is different in cows, where it is localized near the cortex of immature oocytes and absent from the cortical region in matured oocytes and two-cell embryos (Payne \& Schatten 2003). Despite the fact that CRT contains the ER-retrieval sequence KDEL at its C-terminus, it can escape the ER and has been identified on the extracellular surface of a wide variety of cell types (Xiao et al. 1999, Goicoechea et al. 2000) including the surface of the egg plasma membrane and the perivitelline space in mice (Tutuncu et al. 2004). The variations observed in CRT localization might be related to the different antibodies employed in the cited studies detecting $\mathrm{Ca}^{2+}$-bound or $\mathrm{Ca}^{2+}$-free CRT (Balakier et al. 2002) and different CRT isoforms (Zhu et al. 1997). It is also important to bear in mind that the available information comes exclusively from confocal microscopy studies and not from TEM observations.

This study, where both confocal microscopy and TEM were used, shows that CRT is released from subcortical secretory vesicles after oocyte activation and fertilization. CRT colocalized to some extent with PNA lectin as $27.7 \%$ of the pixels in the oocyte's cortical area were concomitantly labeled with PNA (CG contents) and anti-CRT antibody. The TEM observations confirmed

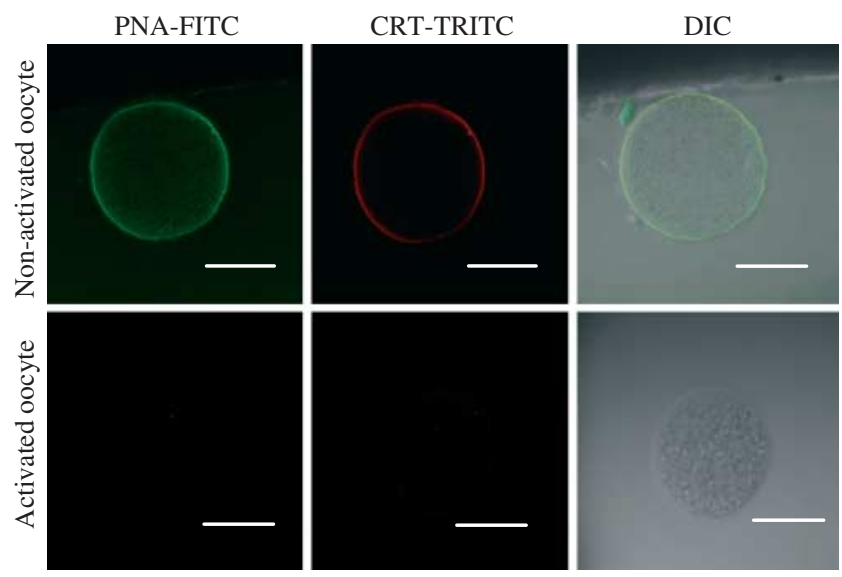

Figure 6 Calreticulin (CRT) and cortical granules (CG) release after activation of ZP-free mature pig oocytes. Immunolocalization of CRT with an anti-CRT antibody with a secondary antibody conjugated to rhodamine (CRT-TRITC) (red) and CG labeled with peanut agglutinin lectin conjugated to fluorescein (PNA-FITC) (green). CRT and CG labeling appear in the cortical area of mature oocytes after ZP digestion by $0.5 \%$ pronase showing. After chemical activation with $6.5 \mu \mathrm{M}$ calcium ionophore for 2 min followed by 30-min incubation, both CRT and CG labeling disappeared. DIC, differential interference contrast. Bar represents $50 \mu \mathrm{m}$. 
Table 1 Calreticulin (CRT) blocks polyspermy at oolemma level. Effect of incubation of pig oocytes with exogenous CRT for 30 min at different concentrations before IVF on further fertilization parameters.

\begin{tabular}{|c|c|c|c|c|c|c|c|c|c|c|c|c|}
\hline \multirow[b]{2}{*}{ CRT (pg/oocyte) } & \multicolumn{6}{|c|}{ ZP-enclosed oocytes } & \multicolumn{6}{|c|}{ ZP-free oocytes } \\
\hline & $n$ & PEN (\%) & $\mathrm{S} / \mathrm{O}$ & MPN $(\%)^{a}$ & $\operatorname{MON}(\%)^{a}$ & SPZ/ZP & $n$ & PEN (\%) & $\mathrm{S} / \mathrm{O}$ & MPN $(\%)^{a}$ & $\operatorname{MON}(\%)^{a}$ & SPZ/OL \\
\hline 0 & 117 & $82.1 \pm 3.6^{*}$ & $2.7 \pm 0.2 *$ & $96.9 \pm 1.8$ & $37.5 \pm 5.0 *$ & $18.6 \pm 0.8^{*}$ & 118 & $77.1 \pm 3.8$ & $3.0 \pm 0.2 *$ & $97.8 \pm 1.5$ & $30.7 \pm 4.8^{*}$ & $11.4 \pm 1.1$ \\
\hline 100 & 107 & $83.2 \pm 3.6^{*}$ & $3.6 \pm 0.2^{\dagger}$ & 100 & $15.7 \pm 3.9^{\dagger}$ & $26.9 \pm 1.1^{\dagger}$ & 120 & $77.5 \pm 3.8$ & $2.7 \pm 0.2^{*}$ & $98.9 \pm 1.1$ & $39.7 \pm 5.1^{*}$ & $10.0 \pm 1.0$ \\
\hline 1000 & 115 & $96.5 \pm 1.7^{\dagger}$ & $3.6 \pm 0.1^{\dagger}$ & $98.2 \pm 1.3$ & $6.4 \pm 2.3^{+}$ & $37.5 \pm 0.5^{\neq}$ & 122 & $77.0 \pm 3.8$ & $2.3 \pm 0.1^{*}$ & 100 & $41.9 \pm 5.1^{*}$ & $12.8 \pm 1.2$ \\
\hline 5000 & 112 & $97.3 \pm 1.5^{\dagger}$ & $4.0 \pm 0.1^{\dagger}$ & 100 & $5.4 \pm 2.2^{+}$ & $39.3 \pm 0.7^{\neq}$ & 121 & $72.7 \pm 4.1$ & $2.0 \pm 0.1^{\dagger}$ & $98.8 \pm 1.1$ & $52.2 \pm 5.3^{\dagger}$ & $10.7 \pm 1.1$ \\
\hline
\end{tabular}

Data represent the mean \pm S.E.M. from four independent experiments. $n$, total number of evaluated oocytes; PEN, penetration; S/O, spermatozoa per penetrated oocyte; MPN, male pronucleus formation; MON, monospermy; SPZ/ZP, sperm bound to zona pellucida in penetrated oocytes; SPZ/OL, sperm attached to oolemma in penetrated oocytes. ${ }^{*,+}+\neq$ Different symbols within each column indicate statistically significant differences $(P<0.05)$. ${ }^{a}$ From penetrated oocytes.

that CRT was not present in all CG, appearing mainly associated with other cortical vesicles. These PDIcontaining vesicles were also released after fertilization. These results are consistent with the observation that mammalian CG contents are heterogeneous, existing as different subpopulations depending on electron density or lectin pattern labeling (Cran 1987, Liu et al. 2003, Jiménez-Movilla et al. 2004). Tutuncu et al. (2004) suggested that CRT is released from mouse egg vesicles distinct from CG as they did not observe colocalization between lens culinaris agglutinin (LCA) lectin and CRT, and the protein was detected in the exudate obtained after oocyte chemical activation. In hamster CG, colocalization between LCA and CRT has been reported (Muñoz-Gotera et al. 2001). The hypothesis that vesicles, distinct from CG and containing different proteins, might be released at fertilization was suggested in mice some time ago (Ducibella et al. 1988) and was suggested in mice with the release of tetraspanin CD9 from mouse eggs (Miyado et al. 2008). These findings open a new field of research and expand the possible mechanisms by which the oocyte modifies its extracellular surface to interact with the spermatozoa.

The exocytosis of CRT during oocyte activation leads us to consider that it may have a role in the block to polyspermy. The amount of CRT in a single egg is unknown, but a mouse egg releases 50-100 pg protein after calcium ionophore activation (Moller \& Wassarman 1989), so our hypothesis was that exocytosed CRT might modify the oolemma, perivitelline space, or the inner part of the ZP due to its chaperoning function, oligosaccharide- and polypeptide-binding. The direct contact of exogenous CRT with the oolemma prevented polyspermy in pigs (ZP-free oocytes), whereas this effect was not observed in ZP-enclosed oocytes, where protein contacted directly with the outer ZP. Effect of exogenous CRT on sperm boar functionality was tested by incubation of sperm with the protein and no detrimental effect was observed (data not shown). Limited information is available about the molecular features of substrate binding to CRT (reviewed by Michalak et al. (2009)) and the protein (extracellular or secreted) adheres to cell surface integrins regulating cellular adhesion (Coppolino et al. 1995, Johnson et al. 2001). Capacitated boar spermatozoa display integrins on their membrane (Barboni et al. 2001), so CRT might be modulating gamete membrane fusion. Furthermore, it is known that porcine oocytes establish a membrane block to polyspermy, which is among the $\mathrm{Ca}^{2+}$-dependent egg activation events (Gardner \& Evans 2006, Gardner et al. 2007). CRT is the major $\mathrm{Ca}^{2+}$-binding chaperone and its fine-tuning together with other $\mathrm{Ca}^{2+}$-binding proteins, such as calsequestrin, plays a crucial role in regulation of $\mathrm{Ca}^{2+}$ transience during oocyte maturation, fertilization, and early embryo development (Balakier et al. 2002). The likely role of CRT regulating porcine gamete fusion at the oolemma level needs further study.

In conclusion, we have demonstrated that CRT locates in cortical vesicles of IVM pig oocytes and that it is exocytosed after oocyte activation, contributing to the membrane block to polyspermy in pigs. Additional studies in different species are necessary to confirm whether this is an evolutionarily conserved mechanism or it is a particular consequence of porcine gamete interactions.

\section{Supplementary data}

This is linked to the online version of the paper at http://dx.doi. org/10.1530/REP-13-0454.

\section{Declaration of interest}

The authors declare that there is no conflict of interest that could be perceived as prejudicing the impartiality of the research reported.

\section{Funding}

This work was supported by MEC and FEDER (grant AGL2009-12512-C02-01) and MINECO (grant AGL201240180-C03-01-02).

\section{Acknowledgements}

The authors thank Juan A Carvajal and Soledad Rodríguez for their excellent technical assistance; Dalland SA farm 
for providing the boar semen samples; the staff of the slaughterhouse El Pozo for supplying the ovaries; and Dr Fara Saez (SAI, University of Murcia) for the image analysis. They also thank Prof. Ronald HF Hunter and Prof. William Holt who carefully read the manuscript and provided us with constructive comments and help with English.

\section{References}

Balakier H, Dziak E, Sojecki A, Librach C, Michalak M \& Opas M 2002 Calcium-binding proteins and calcium-release channels in human maturing oocytes, pronuclear zygotes and early preimplantation embryos. Human Reproduction 17 2938-2947. (doi:10.1093/humrep/17. 11.2938)

Barboni B, Lucidi P, Mattioli M \& Berardinelli P 2001 VLA-6 integrin distribution and calcium signalling in capacitated boar sperm. Molecular Reproduction and Development 59 322-329. (doi:10.1002/ mrd.1037)

Burkart AD, Xiong B, Baibakov B, Jiménez-Movilla M \& Dean J 2012 Ovastacin, a cortical granule protease, cleaves ZP2 in the zona pellucida to prevent polyspermy. Journal of Cell Biology 197 37-44. (doi:10.1083/ jcb.201112094)

Coppolino M, Leung-Hagesteijn C, Dedhar S \& Wilkins J 1995 Inducible interaction of integrin $\alpha 2 \beta 1$ with calreticulin. Dependence on the activation state of the integrin. Journal of Biological Chemistry 270 23132-23138. (doi:10.1074/jbc.270.39.23132)

Coy P \& Avilés $\mathbf{M} 2010$ What controls polyspermy in mammals, the oviduct or the oocyte? Biological Reviews 85 593-605.

Cran DG 1987 The distribution of organelles in mammalian oocytes following centrifugation prior to injection of foreign DNA. Gamete Research 18 67-76. (doi:10.1002/mrd.1120180108)

Ducibella T, Rangarajan S \& Anderson E 1988 The development of mouse oocyte cortical reaction competence is accompanied by major changes in cortical vesicles and not cortical granule depth. Developmental Biology 130 789-792. (doi:10.1016/0012-1606(88)90368-5)

Ellederova Z, Halada P, Man P, Kubelka M, Motlik J \& Kovarova H 2004 Protein patterns of pig oocytes during in vitro maturation. Biology of Reproduction 71 1533-1539. (doi:10.1095/biolreprod.104.030304)

Funahashi H, Cantley TC \& Day BN 1997 Synchronization of meiosis in porcine oocytes by exposure to dibutyryl cyclic adenosine monophosphate improves developmental competence following in vitro fertilization. Biology of Reproduction 57 49-53. (doi:10.1095/biolreprod57.1.49)

Gardner AJ \& Evans JP 2006 Mammalian membrane block to polyspermy: new insights into how mammalian eggs prevent fertilisation by multiple sperm. Reproduction, Fertility, and Development 18 53-61. (doi:10.1071/RD05122)

Gardner AJ, Williams CJ \& Evans JP 2007 Establishment of the mammalian membrane block to polyspermy: evidence for calcium-dependent and -independent regulation. Reproduction 133 383-393. (doi:10.1530/REP06-0304)

Goicoechea S, Orr AW, Pallero MA, Eggleton P \& Murphy-Ullrich JE 2000 Thrombospondin mediates focal adhesion disassembly through interactions with cell surface calreticulin. Journal of Biological Chemistry 275 36358-36368. (doi:10.1074/jbc.M005951200)

Gold LI, Eggleton P, Sweetwyne MT, Van Duyn LB, Greives MR, Naylor SM, Michalak M \& Murphy-Ullrich JE 2010 Calreticulin: non-endoplasmic reticulum functions in physiology and disease. FASEB Journal $\mathbf{2 4}$ 665-683. (doi:10.1096/fj.09-145482)

Gross VS, Wessel G, Florman HM \& Ducibella T 2000 A monoclonal antibody that recognizes mammalian cortical granules and a 32-kilodalton protein in mouse eggs. Biology of Reproduction 63 575-581. (doi:10.1095/biolreprod63.2.575)

Gulyas BJ \& Schmell ED 1980 Ovoperoxidase activity in ionophore-treated mouse eggs. I. Electron microscopic localization. Gamete Research 3 267-277. (doi:10.1002/mrd.1120030309)

Hoodbhoy T \& Talbot P 2001 Characterization, fate, and function of hamster cortical granule components. Molecular Reproduction and Development 58 223-235. (doi:10.1002/1098-2795(200102)58:2<223:: AID-MRD12 > 3.0.CO;2-9)
Hoodbhoy T, Carroll EJ Jr \& Talbot P 2000 Relationship between p62 and p56, two proteins of the mammalian cortical granule envelope, and hyalin, the major component of the echinoderm hyaline layer, in hamsters. Biology of Reproduction 62 979-987. (doi:10.1095/biolreprod62.4.979)

Hoodbhoy T, Dandekar P, Calarco P \& Talbot P 2001 P62/p56 are cortical granule proteins that contribute to formation of the cortical granule envelope and play a role in mammalian preimplantation development. Molecular Reproduction and Development 59 78-89. (doi:10.1002/mrd. 1009)

Jiménez-Movilla $M$, Avilés $M$, Gómez-Torres $M J$, Fernández-Colom PJ, Castells MT, de Juan J, Romeu A \& Ballesta J 2004 Carbohydrate analysis of the zona pellucida and cortical granules of human oocytes by means of ultrastructural cytochemistry. Human Reproduction 19 1842-1855. (doi:10.1093/humrep/deh311)

Johnson S, Michalak M, Opas M \& Eggleton P 2001 The ins and outs of calreticulin: from the ER lumen to the extracellular space. Trends in Cell Biology 11 122-129. (doi:10.1016/S0962-8924(01)01926-2)

Lee SH, Ahuja KK, Gilburt DJ \& Whittingham DG 1988 The appearance of glycoconjugates associated with cortical granule release during mouse fertilization. Development 102 595-604.

Liu M, Sims D, Calarco P \& Talbot P 2003 Biochemical heterogeneity, migration, and pre-fertilization release of mouse oocyte cortical granules. Reproductive Biology and Endocrinology 1 77. (doi:10.1186/ 1477-7827-1-77)

Liu M, Oh A, Calarco P, Yamada M, Coonrod SA \& Talbot P 2005 Peptidylarginine deiminase (PAD) is a mouse cortical granule protein that plays a role in preimplantation embryonic development. Reproductive Biology and Endocrinology 3 42. (doi:10.1186/1477-7827-3-42)

Michalak M, Groenendyk J, Szabo E, Gold LI \& Opas M 2009 Calreticulin, a multi-process calcium-buffering chaperone of the endoplasmic reticulum. Biochemical Journal 417 651-666. (doi:10.1042/ BJ20081847)

Miller DJ, Gong X, Decker G \& Shur BD 1993 Egg cortical granule $\mathrm{N}$-acetylglucosaminidase is required for the mouse zona block to polyspermy. Journal of Cell Biology 123 1431-1440. (doi:10.1083/jcb. 123.6.1431)

Miyado K, Yoshida K, Yamagata K, Sakakibara K, Okabe M, Wang X, Miyamoto K, Akutsu H, Kondo T, Takahashi Y et al. 2008 The fusing ability of sperm is bestowed by CD9-containing vesicles released from eggs in mice. PNAS 105 12921-12926. (doi:10.1073/pnas.0710608105)

Moller C \& Wassarman P 1989 Characterization of a proteinase that cleaves zona pellucida glycoprotein ZP2 following activation of mouse eggs. Developmental Biology 132 103-112. (doi:10.1016/0012-1606(89) 90209-1)

Mondéjar I, Grullón LA, García-Vázquez FA, Romar R \& Coy P 2012 Fertilization outcome could be regulated by binding of oviductal plasminogen to oocytes and by releasing of plasminogen activators during interplay between gametes. Fertility and Sterility 97 453-461. (doi:10.1016/j.fertnstert.2011.11.032)

Muñoz-Gotera R, Hernández-González E, Mendoza-Hernández G, Contreras R \& Mújica A 2001 Exocytosis of a 60 kDa protein (calreticulin) from activated hamster oocytes. Molecular Reproduction and Development 60 405-413. (doi:10.1002/mrd.1103)

Ostwald TJ \& MacLennan DH 1974 Isolation of a high affinity calciumbinding protein from sarcoplasmic reticulum. Journal of Biological Chemistry 249 974-979.

Parys JB, McPherson SM, Mathews L, Campbell KP \& Longo FJ 1994 Presence of inositol 1,4,5-trisphosphate receptor, calreticulin, and calsequestrin in eggs of sea urchins and Xenopus laevis. Developmental Biology 161 466-476. (doi:10.1006/dbio.1994.1045)

Payne C \& Schatten G 2003 Golgi dynamics during meiosis are distinct from mitosis and are coupled to endoplasmic reticulum dynamics until fertilization. Developmental Biology 264 50-63. (doi:10.1016/j.ydbio. 2003.08.004)

Pierce KE, Grunvald EL, Schultz RM \& Kopf GS 1992 Temporal pattern of synthesis of the mouse cortical granule protein, p75, during oocyte growth and maturation. Developmental Biology 152 145-151. (doi:10.1016/0012-1606(92)90164-C)

Rath D \& Niemann H 1997 In vitro fertilization of porcine oocytes with fresh and frozen-thawed ejaculated or frozen-thawed epididymal semen obtained from identical boars. Theriogenology 47 785-793. (doi:10.1016/S0093-691X(97)00034-4) 
Rekkas CA, Besenfelder U, Havlicek V, Vainas E \& Brem G 2002 Plasminogen activator activity in cortical granules of bovine oocytes during in vitro maturation. Theriogenology 57 1897-1905. (doi:10.1016/ S0093-691X(02)00666-0)

Romar R, Coy P, Gadea J \& Rath D 2005 Effect of oviductal and cumulus cells on zona pellucida and cortical granules of porcine oocytes fertilized in vitro with epididymal spermatozoa. Animal Reproduction Science $\mathbf{8 5}$ 287-300. (doi:10.1016/j.anireprosci.2004.04.040)

Romar R, Coy P \& Rath D 2012 Maturation conditions and boar affect timing of cortical reaction in porcine oocytes. Theriogenology $\mathbf{7 8}$ 1126-1139. (doi:10.1016/j.theriogenology.2012.05.009)

Tawia SA \& Lopata A 1992 The fertilization and development of mouse oocytes following cortical granule discharge in the presence of a protease inhibitor. Human Reproduction 7 1004-1009.

Tutuncu L, Stein P, Ord TS, Jorgez CJ \& Williams CJ 2004 Calreticulin on the mouse egg surface mediates transmembrane signaling linked to cell cycle resumption. Developmental Biology 270 246-260. (doi:10.1016/ j.ydbio.2004.02.008)

Wessel GM, Brooks JM, Green E, Haley S, Voronina E, Wong J, Zaydfudim V \& Conner S 2001 The biology of cortical granules. International Review of Cytology 209 117-206.

Xiao G, Chung TF, Pyun HY, Fine RE \& Johnson RJ 1999 KDEL proteins are found on the surface of NG108-15 cells. Molecular Brain Research 72 121-128. (doi:10.1016/S0169-328X(99)00188-6)

Yoshida M, Cran DG \& Pursel VG 1993 Confocal and fluorescence microscopic study using lectins of the distribution of cortical granules during the maturation and fertilization of pig oocytes. Molecular Reproduction and Development 36 462-468. (doi:10.1002/mrd. 1080360409)

Zhang X, Rutledge J, Khamsi F \& Armstrong DT 1992 Release of tissue-type plasminogen activator by activated rat eggs and its possible role in the zona reaction. Molecular Reproduction and Development 32 28-32. (doi:10.1002/mrd.1080320106)

Zhang DX, Li XP, Sun SC, Shen XH, Cui XS \& Kim NH 2010 Involvement of ER-calreticulin- $\mathrm{Ca}^{2+}$ signaling in the regulation of porcine oocyte meiotic maturation and maternal gene expression. Molecular Reproduction and Development 77 462-471. (doi:10.1002/mrd.21166)

Zhu Q, Zelinka P, White T \& Tanzer ML 1997 Calreticulin-integrin bidirectional signaling complex. Biochemical and Biophysical Research Communications 232 354-358. (doi:10.1006/bbrc.1997.6195)

Zinchuk V \& Zinchuk O 2008 Quantitative colocalization analysis of confocal fluorescence microscopy images. Current Protocols in Cell Biology Chapter 4 Unit 4.19.

Received 16 September 2013

First decision 7 October 2013

Revised manuscript received 18 December 2013

Accepted 7 January 2014 